\title{
Associações entre a autoeficácia docente e a utilização do Objective Structured Clinical Examination na educação médica
}

\section{Associations between teacher self-efficacy and the use of Objective Structured Clinical Examination in medical education}

\author{
Rebeca Carvalho Bressa' (D) | rebecabressa@gmail.com \\ Camélia Santina Murgo' (1D) camelia@unoeste.br \\ Bárbara Cristina Soares Sena² (D) barbara_kristina08@hotmail.com
}

\section{RESUMO}

Introdução: A inserção de novas metodologias e avaliações na área da educação médica tem indicado a necessidade de compreender a percepção dos docentes sobre sua própria capacidade de utilizá-las adequadamente.

Objetivo: Com base nisso, este estudo buscou investigar as possíveis associações entre a autoeficácia docente e o uso do OSCE.

Método: Utilizaram-se a Escala de Autoeficácia do Professor, a Escala sobre Fontes de Autoeficácia e um questionário de caracterização. Participaram 47 docentes de Medicina de uma universidade privada, de ambos os sexos, com idade entre 31 e 78 anos.

Resultados: Os resultados indicaram que os fatores persuasão social e aprendizagem vicária foram os mais endossados, sugerindo que essas fontes são as de maior interferência na formação de crenças dos participantes. Houve apenas uma correlação positiva e com significância estatística, com magnitude fraca, estabelecida entre eficácia na intencionalidade da ação e aprendizagem vicária. As demais correlações encontradas se demonstraram estatisticamente em sentido negativo e com magnitudes moderadas.

Conclusões: Os docentes concordantes com algumas características importantes sobre o método OSCE apresentaram maiores níveis de autoeficácia, e isso significa que os profissionais com alto nível de perseverança, superação, confiança e resiliência são mais comprometidos com o ensino, a pesquisa e a assistência estudantil.

Palavras-chave: Autoeficácia Docente; Educação Médica; OSCE.

\section{ABSTRACT}

Introduction: The inclusion of new methodologies and assessments in the medical education area has indicated the need to understand teachers' perception of their own ability to use them adequately.

Objective: Thus, the present study aimed to investigate possible associations between teacher self-efficacy and the use of OSCE.

Method: The Teacher Self-efficacy Scale and Self-Efficacy Sources Scale and a characterization questionnaire were used. Forty-seven medical teachers from a private university, of both genders, aged between 31 and 78 years, participated in the study.

Results: The results indicated that the factors Social Persuasion and Vicarious Learning were the most endorsed, suggesting that these sources are the ones that interfere the most in the formation of the participants' beliefs. There was only one positive and statistically significant correlation, with a weak magnitude, established between the Intentionality of Action Efficacy and Vicarious Learning. The other identified correlations were statistically negative and of moderate magnitude.

Conclusions: The teachers who agreed with some important characteristics about the OSCE method also showed higher levels of self-efficacy. Therefore, professionals with greater perseverance, resilience and confidence, have also been more committed to teaching, research and student assistance.

Keywords: Teacher Self-Efficacy; Medical Education; OSCE.

1 Universidade do Oeste Paulista, Presidente Prudente, São Paulo, Brasil.

${ }^{2}$ Universidade Federal de São Carlos, São Carlos, São Paulo, Brasil.

Editora-chefe: Daniela Chiesa

Editor associado: Roberto Zonato Esteves

Recebido em 01/05/20; Aceito em 02/11/20.

Avaliado pelo processo de double blind review. 


\section{INTRODUÇÃO}

Desde o final do século XX, a educação médica encontrase em constante evolução que ocasionou, ao longo dos anos, diversas mudanças e atualizações na área. Em torno de 1910, o Relatório Flexner tentou estabelecer normas e diretrizes para o ensino médico nos Estados Unidos e no Canadá, e o paradigma flexneriano ficou reconhecido e foi utilizado como base para o ensino médico mundial. Entretanto, com o passar dos anos, o modelo acabou sendo visto como sinônimo de uma educação médica mecanicista, biologista, individualista, especialista e tecnicista'.

No final do século $X X$, essas críticas e discussões levam a medicina a uma aproximação da saúde coletiva ${ }^{2}$. Surge então, em 1970, um novo modelo conhecido como paradigma da medicina integral, que foi reforçado na Conferência Internacional da Organização Mundial da Saúde, em Alma-Ata, no Cazaquistão, em 1978. A partir disso, o conceito de medicina comunitária e sua importância no processo de saúde-doença desencadearam mudanças na maneira de ensinar e na forma de exercer a profissão. Os médicos formados deveriam atender às expectativas sociais, ter habilidades mais específicas, como técnicas de comunicação e posição de liderança, atuar como um profissional generalista e conhecer a atenção primária à saúde ${ }^{3,4}$.

Além das mudanças de paradigma, o ensino médico passa por modificações no processo de ensino e aprendizagem, o que corrobora os novos conceitos vistos na área da educação em geral. De acordo com Marco Antonio Moreira ${ }^{5}$, No contexto educativo, hoje quase não se fala mais em estímulo [...]. Atualmente as palavras de ordem são aprendizagem significativa, mudança conceitual e ensino centrado no aluno". O estudante torna-se autônomo na sua aprendizagem, e novas metodologias tentam auxiliá-lo a construir os próprios caminhos para atingir seu potencial cognitivo ${ }^{6}$.

Diante desses fatores e da necessidade de uma formação prática na área médica, desde a década de 1960, novas metodologias em diversas universidades do mundo passaram a colocar o estudante no centro do processo de aprendizagem ${ }^{7}$. Para atender a essa demanda, houve a inserção de um conjunto de inovações educacionais, como a simulação, que vem tendo um destaque, principalmente, na área médica, como dispositivos tecnológicos e didáticos que auxiliam o processo de ensino-aprendizagem ${ }^{8}$.

A esse respeito, vale esclarecer que existem vários tipos de simuladores e simulações, desde manequins mais simples até robôs que simulam situações específicas de alta-fidelidade realística ${ }^{9,10}$. Na simulação, é possível criar uma situaçãoproblema com atores ou pacientes padronizados e avaliar tarefas específicas em diversas áreas. Com essa flexibilidade, a simulação pode chegar próximo ao real e garantir a observação do aluno em um ambiente seguro, onde o erro pode ser praticado e contribuir para o aprendizado significativo sem prejuízo ao doente ${ }^{11}$.

A avaliação de competências clínicas é, assim como o ensino prático e significativo, uma necessidade na educação médica atual, pois busca verificar a efetividade da aprendizagem no ato profissional, e as tecnologias e os instrumentos pedagógicos mais ativos podem auxiliar na formação dos futuros profissionais ${ }^{12}$. Nessa direção, a Pirâmide de Miller é o instrumento mais utilizado no ensino médico, pois evita que o professor se restrinja apenas ao conhecimento teórico e o estimula a desenvolver o "saber fazer" no processo avaliativo de competências e habilidades ${ }^{13}$.

Com base no desenvolvimento dessas habilitações, a metodologia Objective Structured Clinical Examination - OSCE (Exame Clínico Objetivo Estruturado) pode orientar, de forma mais adequada, esse "saber demonstrar" de que o estudante de Medicina necessita durante o curso. A simulação e a devolutiva ajudam o docente a construir com os discentes o conhecimento e as habilidades profissionais necessários de forma conjunta e mais simultânea ${ }^{14}$. O OSCE é um dos melhores e mais confiáveis métodos de avaliação de competências na educação médica. A primeira experiência desse método foi descrita em 1975, e, desde então, o OSCE é um dos métodos avaliativos mais difundidos pelo mundo. Por ser padronizado e estruturado, OSCE permite que o estudante aprenda com os próprios erros e acertos ao longo do processo de aprendizagem e, por isso, é considerado uma avaliação formativa ${ }^{15,16}$.

O professor-examinador no OSCE é peça fundamental para a aprendizagem, pois a ele competem, de acordo com sua especialidade, as tarefas de verificar a habilidade e a competência e corrigir imperfeições no processo de trabalho do discente ${ }^{17}$. Diversos elementos podem contribuir para efetividade desse tipo de avaliação, não somente o conhecimento técnico do método, mas também a crença do professor que a executa. Portanto, assim como preconiza Bandura ${ }^{18}$, os pensamentos, os sentimentos e as crenças interferem na maneira como uma pessoa se comportará diante de determinada ação.

No contexto educacional, especificamente, podemse considerar as crenças de autoeficácia docente, que é o julgamento do professor sobre a própria capacidade de organizar e alcançar, de forma significativa, o envolvimento e a aprendizagem dos seus estudantes, mesmo diante da diversidade encontrada em sala de aula ${ }^{19}$. Essas crenças são constituídas de quatro fontes de formação, a saber: experiência direta, aprendizagem vicária, persuasão social e estado físico e emocional ${ }^{20,21}$. A experiência direta ou de domínio é a fonte mais efetiva para a criação de forte sensação de autoeficácia, pois se relaciona aos sucessos obtidos com a experiência e vivência 
pessoais de forma direta ${ }^{22}$. As experiências vicárias referemse à observação de modelos sociais com que o indivíduo se identifica. Já a persuasão social ocorre por meio dos feedbacks recebidos de pessoas que tenham credibilidade entre si, ou seja, as pessoas tendem a utilizar exemplos e escutar, de forma mais significativa, outros indivíduos que sejam importantes e confiáveis para elas. Por fim, os estados fisiológicos se configuram como as reações físicas e emocionais perante as demandas, como sudorese, dor no estômago, ansiedade, estresse, entre outras ${ }^{22}$.

Nessa conjuntura, verificar as crenças de autoeficácia pode contribuir para a compreensão da atividade docente na execução do OSCE e suas relações com a aprendizagem significativa. Entender as crenças do professor e como isso interfere na aplicação do OSCE pode ser fundamental no exercício da docência e nas atuais transformações pelas quais a educação médica tem passado.

Ao relacionar o comportamento ao desempenho do estudante, o docente não pode desconsiderar a dimensão emocional no processo de ensino-aprendizagem ${ }^{23}$. E, dessa forma, o professor também deverá se avaliar, pois as relações humanas não são unilaterais. É necessário estimular uma cultura reflexiva da ação docente avaliando a estrutura pessoal desse profissional, seus sentimentos e anseios e, principalmente, suas crenças $^{24,25}$ para fortificar processos pedagógicos como o OSCE. Quanto maiores forem a percepção e o entendimento do docente sobre as próprias práticas educacionais, os sentimentos e as crenças, mais facilmente ele evitará reações que potencializem a angústia e prejudiquem o seu papel de educador ${ }^{26}$.

Segundo laochite ${ }^{27}$, "tão importante quanto os futuros professores adquirirem as habilidades, as competências e os saberes necessários à docência, é que estes acreditem que serão capazes de exercer com algum êxito, suas funções como professores". Um importante aspecto a ser esclarecido, quando se discute sobre a autoeficácia do professor, diz respeito ao papel das crenças de autoeficácia na atuação desse profissional: "Essas crenças influenciam se as pessoas pensam de forma pessimista ou otimista e de maneira que ajudem ou atrapalhem o indivíduo" ${ }^{28}$.

Isso posto, considera-se que as crenças de autoeficácia podem interferir no processo de ensino-aprendizagem não só vinculadas ao aprendizado discente, mas também sob a ação docente. Portanto, somente um professor que reflete constantemente sobre suas ações pedagógicas e avalia, monitora e regula suas crenças de autoeficácia é capaz de deflagrar em seus alunos um processo de aprendizagem significativa ${ }^{19}$. Além disso, um docente com uma percepção mais elevada de autoeficácia pode ter maior aceitação e melhor utilização de técnicas e instrumentos de formação, como o uso do OSCE como processo avaliativo na educação médica.

Há, na literatura, uma escassez de estudos que se proponham a identificar e relacionar a autoeficácia do professor de Medicina. O que se indica é que ser exposto a bons docentes pode melhorar a apreensão dos conhecimentos e o desempenho clínico dos estudantes de Medicina. Mas, ainda assim, poucos estudos têm se dedicado a compreender os mecanismos psicológicos relacionados à prática docente ${ }^{29}$. Portanto, de acordo com Dybowski et al. ${ }^{29}$, a qualidade do ensino pode se beneficiar de professores que são autoeficazes e capazes de detectar e fomentar as competências dos estudantes.

Diante disso, o objetivo geral desta pesquisa é analisar a relação entre a autoeficácia docente e a efetividade na aplicação do OSCE como instrumento de avaliação formativa. Para tanto, foram verificadas as relações da autoeficácia com relação ao manejo de sala de aula e à intencionalidade da ação docente, bem como se identificaram as associações entre a aplicação do OSCE e as crenças de autoeficácia docente.

\section{MÉTODO}

\section{Participantes}

Participaram deste estudo 47 docentes do curso de Medicina de uma universidade privada do interior do estado de São Paulo, sendo 55,3\% $(n=26)$ do sexo masculino e $44,7 \%(n=21)$ do sexo feminino, com idade variando de 31 a 78 anos $(M=44,62 ; D P=10,35)$. É importante destacar que, desses profissionais, 91,5\% $(n=43)$ são médicos, e 8,5 \% ( $=4$ ), enfermeiros. O tempo de experiência na docência varia de um a 35 anos, com média de 11,5 anos ( $M=11,51$; DP = $7,70)$ de tempo de profissão. Já em relação à titulação, 63,8\% ( $n=30$ ) mencionaram a pós-graduação lato sensu, enquanto um número menor declarou ter mestrado $(29,8 \% ; n=14)$ e doutorado $(6,4 \% ; n=3)$.

Por fim, sobre a área de atuação/especialidade, verificouse maior concentração em clínica médica $(31,9 \% ; n=15)$, emergência (19,1\%; $n=9)$, clínica cirúrgica $(14,9 \%, n=7)$, saúde coletiva e pediatria $(12,8 \%, n=6$, em cada uma), e ginecologia e obstetrícia obtiveram a menor frequência: $8,5 \%, n=4$.

\section{Instrumentos}

Para a coleta de dados, utilizaram-se os seguintes instrumentos:

- Escala de Autoeficácia do Professor (EAEP) ${ }^{30}$ : Trata de uma adaptação da Ohio State TeacherEfficacy Scale $e^{31}$ que avalia a autoeficácia do professor em relação ao manejo de sala de aula e a intencionalidade da ação docente. A escala possui dois fatores (subescalas): 1. intencionalidade na ação docente, que representa a capacidade do professor de 
mobilizar o estudante para realização da tarefa e de mediação do ensino $(a=0,87)$ e 2 . eficácia no manejo da classe, referente à crença docente para gerenciar os múltiplos aspectos do cotidiano da aula e lidar com eles $(a=0,76)$. A confiabilidade da escala total foi de 0,91 ((alfa de Cronbach).

- Escala sobre Fontes de Autoeficácia (EFAE) ${ }^{27}$ : Avalia a interferência das quatro fontes de formação das crenças de autoeficácia. Esse instrumento é composto por itens que referenciam as quatro fontes de autoeficácia propostas por Bandura ${ }^{32}$. Os itens representam as fontes de experiência direta $(5$, 12 e 13; $a=0,36)$, experiências vicárias (1, 2 e 6; $a=$ 0,54), persuasão social $(3,10,11,14$ e 15; $a=0,62)$ e, por último, estados fisiológico e afetivos $(4,7,8,9$ e $16 ; a=0,82$ ). A confiabilidade da escala total foi de 0,83 (alfa de Cronbach).

- Questionário de Caracterização do Participante $(Q C P):$ Nesse instrumento, além da descrição sociodemográfica, incluíram-se questões assertivas referentes às principais características de atividades de simulação, como o OSCE, conforme revisão sistemática de Issenberg et al. ${ }^{33}$, e às respectivas percepções docentes sobre o assunto e o contexto de trabalho. Das 26 questões que compuseram o QCP, as dez primeiras abordaram aspectos relacionados aos dados sociodemográficos, à formação e à área de atuação do participante, e as outras 16 foram estruturadas por meio de uma escala do tipo Likert (cinco pontos), que vai de "discordo totalmente" a "concordo totalmente". O instrumento apresenta dois fatores: bem-estar docente $(a=0,68)$ e principais características $(a=0,73)$. A confiabilidade da escala total foi de 0,81 (alfa de Cronbach).

\section{Análise de dados}

Os dados foram analisados por meio do software Statistical Package for the Social Sciences (SPSS), versão 21, com estatísticas descritivas para caracterização da amostra e das médias dos participantes nos instrumentos. Posteriormente, ocorreram as estatísticas inferenciais com correlação de Pearson entre os fatores dos instrumentos. Para interpretação dos dados correlacionais, utilizaram-se os parâmetros de Dancey e Reid $^{34}$, nos quais os coeficientes de correlação com valor 0 são considerados nulos; entre 0,10 e 0,30, fracos; a partir de 0,40 e até 0,60, moderados; de 0,70 a 0,90, fortes; e correlações de 1 são perfeitas. Complementarmente, realizaram-se as análises dos índices de confiabilidade dos fatores das escalas por meio do alfa de Cronbach para a amostra deste estudo.

\section{RESULTADOS}

Com o objetivo de analisar a relação entre a autoeficácia docente e efetividade na aplicação do OSCE como instrumento de avaliação formativa, empregaram-se inicialmente as estatísticas descritivas das pontuações obtidas no QCP e as afirmativas direcionadas para a percepção docente sobre o contexto e o OSCE, numa subdivisão do QCP: percepção docente sobre o contexto (PDCONTEXTO) e percepção docente sobre o OSCE (PDOSCE).

Realizaram-se também estatísticas descritivas considerando as pontuações médias nos fatores da EAEP, da EFAE e do QCP, subdivisão: PDCONTEXTO e PDOSCE.

De acordo com a Tabela 1, na EAEP, as maiores médias foram em relação ao fator eficácia na intencionalidade da ação, o que já foi demonstrado em outras pesquisas, como as de laochite ${ }^{27}$ e Rocha ${ }^{35}$. Já na EFAE, o fator persuasão social foi o mais endossado pelos participantes, obtendo a maior média quando comparado com os demais fatores dos instrumentos, o que pode indicar que essa fonte é a de maior interferência na formação de crenças dos professores.

Tabela 1. Estatísticas descritivas dos resultados da EAEP e EFAE e da subdivisão do QCP (PDCONTEXTO, PDOSCE)

\begin{tabular}{|c|c|c|c|c|c|}
\hline Fatores/instrumento & $\mathbf{N}$ & Mínimo & Máximo & M & DP \\
\hline PDCONTEXTO & 47 & 18 & 30 & 25,46 & 2,94 \\
\hline PDOSCE & 47 & 19 & 43 & 36,59 & 4,48 \\
\hline Eficácia no manejo da classe & 47 & 37 & 60 & 48,93 & 5,25 \\
\hline Eficácia na intencionalidade da ação & 47 & 50 & 84 & 69,36 & 7,66 \\
\hline Experiência direta & 47 & 8 & 18 & 14,91 & 2,43 \\
\hline Aprendizagem vicária & 47 & 9 & 18 & 15,14 & 2,4 \\
\hline Persuasão social & 47 & 14 & 30 & 25,51 & 3,61 \\
\hline Estados fisiológicos & 47 & 6 & 28 & 17,17 & 6,68 \\
\hline
\end{tabular}


Nas fontes experiência direta $(M=14,91)$ e a aprendizagem vicária $(M=15,14)$, os professores apresentaram as médias mais baixas. A principal hipótese para esse resultado está relacionada ao fato de os professores da instituição de ensino pesquisada terem pouco tempo de experiência na aplicabilidade do OSCE, haja vista que o instrumento só foi implantado em julho de 2015 e tornou-se efetivo em todas as áreas clínicas básicas apenas no final de 2016. Apesar de 27 professores $(57,4 \%)$ terem mais de dez anos de experiência docente e oito (17\%) mais de 20 anos, talvez os subsídios fundamentais de prática nesse tipo de avaliação e em uma metodologia mais ativa de aprendizagem ainda não tenham se formado nesse grupo de profissionais.

Em seguida, buscou-se verificar as correlações entre os itens da PDCONTEXTO e da PDOSCE com a EAEP, conforme indica a Tabela 2.

Observa-se que, para os fatores PDCONTEXTO e PDOSCE, não foram encontradas correlações totais significativas com nenhum dos fatores da EAEP. O que se observa são relações de algumas assertivas, sendo de forma isolada, em sentido positivo e com magnitude fraca. O item Q13 ("Sente-se preparado(a) para exercer sua atividade docente"), por exemplo, apresentou significância positiva com as duas variáveis de Autoeficacia Docente (manejo de sala de aula e intencionalidade na ação).

Encontraram-se também relações entre outros itens e variáveis: o item Q18 ("Dificuldade da simulação é importante") mostrou significância positiva com a eficácia do manejo da classe, e os itens Q21 ("OSCE prepara o aluno para a prática profissional") e Q25 ("OSCE proporciona maior segurança no trabalho docente e maior eficácia no aprendizado discente") se relacionaram positivamente com a eficácia na intencionalidade da ação.

$\mathrm{Na}$ sequência, buscou-se verificar as relações entre a percepção (PDCONTEXTO e PDOSCE) e os fatores de fontes de autoeficácia. Observa-se, na Tabela 3, que, em relação às fontes de experiência direta e estados fisiológicos e afetivos, não foram encontradas correlações com significância estatística. Já para as fontes de aprendizagem vicária e persuasão social, as correlações foram estatisticamente significativas, em sentido positivo e com magnitude variando de fraca a moderada.

Tabela 2. Correlações entre os fatores de percepção (PDCONTEXTO e PDOSCE) e a Escala de Autoeficácia do Professor (EAEP)

\section{PDCONTEXTO:}

Eficácia no manejo da classe

Eficácia na intencionalidade da ação

\begin{tabular}{l} 
Q11 - Expressão livre de ideias. \\
\hline Q12 - Apoio dos colegas. \\
\hline Q13 - Sente-se preparado para exercer a docência. \\
\hline Q14 - Sente-se satisfeito como docente.
\end{tabular}

TOTAL 0,01

$-0,26$ $-0,03$

$0,41^{* *}$

$-0,22$

PDOSCE:

Q15 - O feedback é importante.

0,18

$0,40^{* *}$

Q16 - Prática sistematizada é primordial para eficácia.

0,2

0,25

Q17 - Promove integração de disciplinas.

$0,29 *$

Q18 - Dificuldade da simulação é importante.

0,2

0,25

Q19 - É mais eficaz para desenvolver e corrigir habilidades do que a prática real isolada.

$0,02-0,02$

$0,43^{* *}$

$0,29^{*}$

0,14

0,16

Q20 - Ambiente controlado sem consequência adversa.

Q21 - Prepara o aluno para a prática profissional.

Q22 - Estudante é ativo.

Q23 - Objetivos específicos e estudante domina habilidade.

\section{$-0,22$}

0,11

$-0,23$

$-0,12$

$-0,13$

0,21

0,23

0,09

$-0,2$

Q24 - Proporciona aprendizagem significativa.

Q25 - Proporciona maior segurança no trabalho

Q26 - Proporciona condições para atualização docente.

TOTAL

${ }^{*} p<0,05 ;{ }^{* *} p<0,01$. 
Tabela 3. Correlações entre os fatores de percepção (PDCONTEXTO e PDOSCE) e a Escala sobre Fontes de Autoeficácia (EFAE)

\begin{tabular}{|c|c|c|c|c|}
\hline PDCONTEXTO: & $\begin{array}{l}\text { Experiência } \\
\text { direta }\end{array}$ & $\begin{array}{l}\text { Aprendizagem } \\
\text { vicária }\end{array}$ & $\begin{array}{l}\text { Persuasão } \\
\text { social }\end{array}$ & $\begin{array}{c}\text { Estados fisiológicos } \\
\text { e afetivos }\end{array}$ \\
\hline Q11 - Expressão livre de ideias. & 0,17 & $0,30^{*}$ & 0,18 & 0,11 \\
\hline Q12 - Apoio dos colegas. & $-0,01$ & $-0,04$ & $-0,05$ & 0,13 \\
\hline $\begin{array}{l}\text { Q13 - Sente-se preparado para exercer a } \\
\text { docência. }\end{array}$ & 0,05 & 0,11 & $-0,06$ & $-0,42 * *$ \\
\hline Q14 - Sente-se satisfeito como docente. & $-0,01$ & 0,16 & 0,16 & $-0,15$ \\
\hline TOTAL & 0,24 & $0,38 * *$ & $0,38 * *$ & $-0,00$ \\
\hline \multicolumn{5}{|l|}{ PDOSCE: } \\
\hline Q15 - O feedback é importante. & 0 & $0,31 *$ & 0,24 & 0 \\
\hline $\begin{array}{l}\text { Q16 - Prática sistematizada é primordial } \\
\text { para eficácia. }\end{array}$ & 0,1 & $0,39 * *$ & $0,49 * *$ & 0,22 \\
\hline Q17 - Promove integração de disciplinas. & $-0,05$ & $-0,03$ & $-0,04$ & $-0,01$ \\
\hline $\begin{array}{l}\text { Q18 - Dificuldade da simulação é } \\
\text { importante. }\end{array}$ & 0,13 & $0,32 *$ & 0,27 & $-0,01$ \\
\hline $\begin{array}{l}\text { Q19 - É mais eficaz para desenvolver e } \\
\text { corrigir habilidades do que a prática real } \\
\text { isolada. }\end{array}$ & 0,14 & $0,46 * *$ & $0,33^{*}$ & 0,1 \\
\hline $\begin{array}{l}\text { Q20 - Ambiente controlado sem } \\
\text { consequência adversa. }\end{array}$ & 0,09 & $-0,23$ & 0 & $0,36^{*}$ \\
\hline $\begin{array}{l}\text { Q21 - Prepara o aluno para a prática } \\
\text { profissional. }\end{array}$ & $0,31 *$ & $0,51 * *$ & $0,41 * *$ & 0,27 \\
\hline Q22 - Estudante é ativo & $-0,04$ & $-0,09$ & 0,13 & 0,24 \\
\hline $\begin{array}{l}\text { Q23 - Objetivos específicos e estudante } \\
\text { domina habilidade. }\end{array}$ & 0,02 & $-0,15$ & $-0,15$ & 0,13 \\
\hline $\begin{array}{l}\text { Q24 - Proporciona aprendizagem } \\
\text { significativa. }\end{array}$ & 0,05 & $-0,08$ & 0,01 & 0,11 \\
\hline $\begin{array}{l}\text { Q25 - Proporciona maior segurança no } \\
\text { trabalho docente e maior eficácia no } \\
\text { aprendizado discente. }\end{array}$ & 0,3 & $0,37 * *$ & $0,51 * *$ & 0,1 \\
\hline $\begin{array}{l}\text { Q26 - Proporciona condições para } \\
\text { atualização docente. }\end{array}$ & 0,26 & $0,36 *$ & $0,44^{* *}$ & 0,02 \\
\hline TOTAL & 0,11 & $0,29 *$ & $0,32 *$ & 0,28 \\
\hline
\end{tabular}

$* * p<0,01$.

As correlações entre os fatores isolados da PDCONTEXTO e da PDOSCE e os da EFAE foram, em sua maioria, nulas ou sem significância estatística. Aquelas que foram estatisticamente significativas variaram entre magnitude fraca a moderada e com sentido positivo, exceto para o item Q13 ("Sente-se preparado para exercer a docência") e o fator estados fisiológicos e afetivos em que a correlação foi negativa. Isso pode significar que quanto mais se sentem preparados para exercer a docência, menos a fonte de estados fisiológicos e afetivas interfere na formação das crenças dos professores do grupo estudado.

Já as relações positivas encontradas, de acordo com as fontes de autoeficácia, foram, por exemplo, a aprendizagem vicária com os itens Q11 ("Expressão livre de ideias"), Q15 ("O feedbackéimportante"), Q16 ("Prática sistematizada é primordial para eficácia"), Q18 ("Dificuldade da simulação é importante"), Q19 ("É mais eficaz para desenvolver e corrigir habilidades do que a prática real isolada"), entre outros; a persuasão social com os itens Q16 ("Prática sistematizada é primordial para eficácia"), Q19 ("É mais eficaz para desenvolver e corrigir habilidades do que a prática real isolada"), Q21 ("Prepara o aluno para a prática profissional"), Q25 ("Proporciona maior segurança no trabalho docente e maior eficácia no aprendizado discente") e Q26 ("Proporciona condições para atualização docente"); e, por fim, a experiência direta com Q21 ("Prepara o aluno para a prática profissional") e estados fisiológicos e afetivos com Q20 ("Ambiente controlado sem consequência adversa").

Por fim, buscou-se as relações entre os tipos de autoeficácia e as fontes, como visto na Tabela 4. 
Tabela 4. Correlações entre fatores da Escala de Autoeficácia do Professor (EAEP) e da Escala sobre Fontes de Autoeficácia (EFAE)

\begin{tabular}{|c|c|c|c|c|}
\hline & Experiência direta & Aprendizagem vicária & Persuasão social & $\begin{array}{c}\text { Estados fisiológicos e } \\
\text { afetivos }\end{array}$ \\
\hline Eficácia no manejo de sala & $-0,11$ & 0,26 & 0,02 & $-0,42^{* *}$ \\
\hline Eficácia na intencionalidade da ação & $-0,07$ & $0,32^{*}$ & 0,04 & $-0,40^{* *}$ \\
\hline
\end{tabular}

${ }^{*} p<0,05 ;{ }^{* *} p<0,01$

A maior parte das correlações foinula ou sem significância estatística. No que concerne às correlações estatisticamente significativas, elas foram em sentido negativo e com magnitudes moderadas entre os fatores de autoeficácia e as fontes de estados fisiológicos e afetivos, indicando que quanto maior é a crença na capacidade de ensinar ou controlar o ambiente acadêmico, menores são as influências de tensões fisiológicas e emocionais no desempenho e na formação dessas crenças. Houve apenas uma correlação positiva e com significância estatística, com magnitude fraca, estabelecida entre eficácia na intencionalidade da ação e aprendizagem vicária, sugerindo que os docentes que acreditam na sua capacidade de mobilizar os estudantes para o processo de ensino-aprendizagem podem ter essas crenças formadas a partir das próprias experiências vividas em sala de aula ou decorrentes das experiências de seu processo formativo como um todo.

\section{DISCUSSÃO}

Os dados indicam inicialmente a importância da eficácia na intencionalidade da ação sobre a percepção de autoeficácia dos docentes, informações que corroboram outros estudos já realizados ${ }^{27,35}$. Conforme observado por Polydoro et al. ${ }^{30}$ na adaptação nacional do instrumento Ohio State Teacher Efficacy Scale ${ }^{31}$, a intencionalidade da ação representa a crença do professor na capacidade de mediar o ensino e mobilizar o estudante para a realização das atividades. Portanto, diz respeito a como o docente pode ajudar seus estudantes a valorizar o processo de ensino-aprendizagem e abarca as ações de acompanhar, incentivar, valorizar e realizar feedback durante o processo de aprendizagem.

Outra fonte que recebeu destaque sobre o julgamento que os professores fazem sobre suas próprias capacidades foi a persuasão social, sendo analisada como a fonte de maior interferência. Segundo Bandura ${ }^{32}$, a persuasão social é uma das fontes mais prevalentes em professores iniciantes, e também há nesse grupo porcentagem alta de docentes sem experiência em qualquer atividade profissional, visto que $20(42,6 \%)$ tinham menos de dez anos de docência, e, destes últimos, dez $(21,3 \%)$ tinham menos de cinco anos de experiência.

Ainda nessa perspectiva, a persuasão social é uma das fontes mais distantes para fortalecer a autoeficácia, pois pode ser limitada no alcance da crença pessoal de forma duradoura. Mas ela poderá reforçar a autoavaliação se houver uma apreciação positiva de que o incentivo esteja dentro dos limites da realidade ${ }^{21}$. Supostamente, os professores participantes desta pesquisa estão sendo incentivados pela instituição de educação superior, mas ainda não adquiriram, de forma adequada, experiência ou conhecimento pedagógico e científico do método avaliativo formativo para fortificar suas crenças. Nesse sentido, como dito por Bandura ${ }^{32}$, o simples apontamento verbal sobre a capacidade de alguém não é o suficiente, pois também é necessário que o ambiente providencie experiências que afirmem a eficácia durante um tempo.

Quanto às associações dos fatores da atuação profissional com a percepção de autoeficácia, observou-se que se sentir preparado para exercer a sua atividade aumentará a crença na capacidade de manejar a sala de aula e manterá a intencionalidade da sua ação. Ou seja, sentir-se preparado para exercer a prática docente fortalece as crenças do professor em gerenciar o cotidiano na sala e mobilizar o estudante para a realização de tarefas. Bandura ${ }^{32}$ explica, de acordo com a Teoria Social Cognitiva, que o interesse pessoal é desenvolvido por causa de autorreações afetivas e dos mecanismos da autoeficácia. Assim, as pessoas mantêm interesse permanente em tarefas ou atividades nas quais se sintam capazes, eficazes e satisfeitas.

Também foram identificadas relações entre a autoeficácia e a utilização do OSCE na prática docente. Portanto, ao perceber que a simulação é parte importante da formação e preparação do estudante e proporciona maior segurança no momento de ensino, o professor de Medicina tem um aumento positivo na eficácia da intencionalidade da sua ação em sala de aula. Esses dados podem indicar algumas possíveis relações entre o uso do OSCE e a autoeficácia, pois demonstram, por exemplo, que o docente que se sente capaz de lidar com o cotidiano na sala de aula e gerenciá-lo acredita que a dificuldade da simulação pode ser uma variável importante para o aprendizado do aluno em um cenário clínico de OSCE. Esses dados também preconizam que, presumivelmente, os professores que têm crenças fortes na sua capacidade de mobilizar os alunos para a realização de tarefas e para o ensino também acreditam que o OSCE prepara o estudante para a prática profissional. E ainda apontam que os professores que mobilizam os alunos para realização de tarefas e para o 
ensino acreditam que o OSCE proporciona maior segurança no trabalho docente e maior eficácia no aprendizado discente.

Sobre sentir-se preparado para exercer a docência e utilizar o OSCE, foram identificadas correlações, ainda que negativas, com os estados fisiológicos e emocionais dos professores. Os estados físicos emocionais, como o estresse, a fadiga, a ansiedade, a tensão, a dor e os estados de humor, são manifestações que podem interferir na percepção de autoeficácia. Isso acontece porque essas sensações afetam diretamente o julgamento que a pessoa tem sobre a sua própria capacidade para realizar uma tarefa ${ }^{21}$. O controle dessas sensações e o enfrentamento delas são condições importantes para se obter sucesso, contribuindo para fortalecer as crenças de autoeficácia ${ }^{36}$. Desse modo, existe uma diminuição desses estados quando a percepção do docente sobre estar preparado está fortalecida.

Como indicado nos resultados, também foram encontradas relações positivas entre as demais fontes de autoeficácia (aprendizagem vicária, persuasão social e experiências diretas) com fatores cotidianos do ensino médico (uso de práticas sistematizadas, simulação como meio eficaz de desenvolver e corrigir habilidades, preparação dos estudantes para a prática médica, entre outras). Com essas relações, observa-se que algumas características do OSCE se repetem e se mostram importantes principalmente nas fontes com significância estatística positiva. Essas características (importância do feedback e da sistematização, eficaz para corrigir habilidades, maior segurança, atualização docente e preparação para a prática profissional) são condizentes com o que recomendam Khan et al. $^{37}$ sobre o OSCE. Os autores descrevem que, apesar de a técnica poder avaliar os três domínios de aprendizagem (cognitivo, afetivo e psicomotor), não é possível testar todos os níveis de dificuldade dentro de cada domínio com o uso de um único OSCE. O OSCE deve ser projetado para avaliar certas competências ou habilidades que não podem ser avaliadas por meio do uso de caneta e papel ou de testes baseados em métodos computadorizados ${ }^{37}$.

No âmbito nacional, outras pesquisas que utilizaram os mesmos instrumentos deste estudo mostraram uma tendência para níveis médios e, frequentemente, elevados de autoeficácia docente em professores no ensino superior ${ }^{27,35,38}$. As hipóteses que justificam o fato de o grupo docente da presente pesquisa ter obtido nível mais mediano de autoeficácia devem ser confirmadas com novas pesquisas e análises qualitativas que abordem as especificidades da docência médica e com o uso de técnicas como o OSCE.

Em relação às médias nas subescalas da EAEP, podese verificar média mais alta no fator intencionalidade da ação docente quando comparado ao fator manejo de classe. Tal diferença também foi encontrada nos estudos realizados por laochite ${ }^{27}$ e Rocha ${ }^{35}$. Dybowski et al. ${ }^{29}$ também utilizaram uma escala de autoeficácia própria desenvolvida em seus estudos, e os resultados apontaram que a qualidade do ensino pode se beneficiar da formação de professores, da capacidade de detectar competências de seus alunos e da melhoria da autoeficácia no ensino médico.

Como postulado por Bandura ${ }^{32}$ e confirmado em vários estudos $^{21,22}$, as principais fontes de autoeficácia constituem experiências diretas, experiências vicárias, persuasão social e interpretação subjetiva dos estados fisiológicos e afetivos durante uma ação. Segundo Dybowski et al..29, a formação de professores deveria se basear nesses princípios para efetivamente melhorar a autoeficácia do ensino médico. Avaliações de progresso como o OSCE podem ser mais confiáveis para a qualidade do ensino e esclarecer as relações entre motivação do ensino, autoeficácia do ensino e qualidade do ensino clínico.

\section{CONCLUSÕES}

O presente estudo teve como objetivo geral analisar a relação entre a autoeficácia docente e a efetividade na aplicação do OSCE como instrumento de avaliação formativa. O estudo encontrou maior número de professores com média e baixa autoeficácia, e a fonte de eficácia mais prevalente foi a persuasão verbal, o que necessita ser confirmado com novas abordagens qualitativas, como grupo focal, entrevista ou mesmo uma pesquisa etnográfica. Evidenciaram-se tendências que precisam ser estudadas e confirmadas também em novas pesquisas com maior número de professores e em diferentes instituições.

Ainda assim, os dados indicam pontos importantes a serem considerados, como a principal observação feita de que docentes concordantes com algumas características importantes sobre o método OSCE também apresentaram maiores níveis de autoeficácia. Logo, trata-se dos profissionais com maior perseverança, superação, confiança e resiliência, qualidades essenciais a um professor comprometido com o ensino, a pesquisa e a assistência. Quanto às limitações do estudo, destaca-se o tamanho pequeno da amostra. Portanto, recomendam-se investigações futuras que ampliem a amostra para diferentes contextos e escolas médicas. Além disso, estudos com uma amostra maior permitiria o uso de outras abordagens para descrever a relação entre os constructos, como a teoria de resposta ao item multidimensional inserida na modelagem de equação estrutural.

Esta investigação pretende contribuir para a divulgação do constructo de autoeficácia na docência médica e aprofundar a técnica OSCE como aprendizagem significativa. Assim, a avaliação pode deixar de ser um "acerto de contas" para se tornar 
uma oportunidade de aprendizado efetivo, diferentemente do que ocorre com a aprendizagem mecânica pura. Rever, de forma persistente, o processo de trabalho docente e instrumentalizar o professor com a seriedade de métodos como o OSCE e constructos como a autoeficácia resultará na formação de um profissional médico mais condizente com as necessidades sociais vigentes e mais humanista.

\section{CONTRIBUIÇÃO DAS AUTORAS}

Rebeca Carvalho Bressa e Camélia Santina Murgo planejaram e executaram o projeto e a coleta de dados, bem como escreveram os resultados e as discussões. Bárbara Cristina Soares Sena escreveu a introdução e fez as revisões necessárias. Todas as autoras contribuíram para a redação do manuscrito.

\section{CONFLITO DE INTERESSES}

Declaramos que não há conflitos de interesse em potencial que possam influenciar o processo de publicação.

\section{FINANCIAMENTO}

Declaramos que não houve financiamento para a realização desta pesquisa.

\section{REFERENCIAS}

1. Amore Filho ED. Ações para a retomada do ensino da humanização nas escolas de medicina: uma revisão sistemática da literatura, 2010-2016 [dissertation]. Belo Horizonte: Universidade José do Rosário Vellano; 2018 [access in 20 oct. 2019]. Available from: http://tede2.unifenas.br:8080/ jspui/handle/jspui/200.

2. Mota A, Schraiber LB, Ayres JRCM. Desenvolvimentismo e preventivismo nas raízes da saúde coletiva: reformas do ensino e criação de escolas médicas e departamentos de medicina preventiva no estado de São Paulo (1948-1967). Interface Comum Saúde Educ. 2018;22(65):337-48. doi: 10.1590/1807-57622017.0810.

3. Torres ACS. Formação médica para a APS: percepções de egressos da UFC [dissertation]. Fortaleza: Universidade Federal do Ceará; 2016 [access in 8 dec. 2019]. Available from: http://www.repositorio.ufc.br/handle/ riufc/22543.

4. Rocha VXM. Reformas na educação médica no Brasil: estudo comparativo entre as diretrizes curriculares nacionais dos cursos de graduação em medicina de 2001 e 2014 [dissertation]. Santos: Universidade Católica de Santos; 2017 [access in 8 dec. 2019]. Available from: http://biblioteca. unisantos.br:8181/handle/tede/4441.

5. Moreira MA. Aprendizagem significativa: um conceito subjacente. Aprendizagem Significativa em Revista. 2011;1(3):25-46 [access in 20 oct. 2019]. Available from: http://www.if.ufrgs.br/asr/artigos/Artigo_ID16/ v1_n3_a2011.pdf.

6. Winters JRF.Tecnologias de informação e comunicação (TICs) na graduação em enfermagem: possibilidades e limites na formação de profissionais críticos-criativos e reflexivos [thesis]. Florianópolis: Universidade Federal de Santa Catarina; 2018 [access in 20 oct. 2014]. Available from: http:// joinville.ifsc.edu.br/ bibliotecajoi/arquivos/thesiss/201312.pdf.

7. Conterno SFR, Lopes RE. Pressupostos pedagógicos das atuais propostas de formação superiorem saúdeno Brasil:origens históricas efundamentosteóricos. Avaliação. 2016;21(3):993-1016. doi: 10.1590/S1414-40772016000300016.

8. Gomez MV, Vieira JE, Scalabrini-Neto A. Análise do perfil do professor da área da saúde que usam a simulação como estratégia didática. Rev Bras Educ Med. 2011;35(2):157-62. doi: 10.1590/S0100-55022011000200003.
9. Ferreira C, Carvalho JM, Carvalho FLQ. Impacto da metodologia de simulação realística, enquanto tecnologia aplicada a educação nos cursos de saúde. STAES, III Seminário de Tecnologias Aplicadas em Educação e Saúde; 8-9 maio 2017; Salvador, Brasil. Salvador: Uneb; 2017. p. 32-40.

10. Kaneko RMU, Lopes MHBM. Cenário em simulação realística em saúde: o que é relevante para a sua elaboração? Rev Esc Enferm USP. 2019;53:e03453. doi: 10.1590/s1980-220x2018015703453.

11. Negri EC, Mazzo A, Martins JCA, Pereira Junior GA, Almeida RGS, Pedersoli CE. Simulação clínica com dramatização: ganhos percebidos por estudantes e profissionais de saúde. Rev Latino-Am Enferm. 2017;25:e2916. doi: 10.1590/1518-8345.1807.2916.

12. Zimmermann MH, Silveira RMCF, Gomes RZ. O professor e a arte de avaliar no ensino médico de uma universidade no Brasil. Rev Bras Educ Med. 2019;43(3):5-15. doi: 10.1590/1981-52712015v43n3rb20180167.

13. Amaral E, Domingues RCL, Bicudo-Zeferino AM. Avaliando competência clínica: o método de avaliação estruturada observacional. Rev Bras Educ Med. 2007;31(3):287-90. doi: 10.1590/50100-55022007000300011.

14. Franco CAGS, Franco RS, Santos VM, Uiema LA, Mendonça NB, Casanova AP, et al. OSCE para competências de comunicação clínica e profissionalismo: relato de experiência e meta-avaliação. Rev Bras Educ Med. 2015;39(3):433-41. doi: 10.1590/1981-52712015v39n3e02832014.

15. Souza RGS. Atributos fundamentais dos procedimentos de avaliação. In: Tibério IFLC, Daud-Gallotti RM, Troncon LEA, Martins MA, organizers. Avaliação prática de habilidades clínicas em medicina. São Paulo: Atheneu; 2012. p. 1-11.

16. Rocha SR, Romão GS, Setúbal MSV, Collares CF, Amaral E. Avaliação de habilidades de comunicação em ambiente simulado na formação médica: conceitos, desafios e possibilidades. Rev Bras Educ Med. 2019;43(1 Supl. 1):236-45. doi: 10.1590/1981-5271v43suplemento1-20190154.

17. Dantas AKC. Uso do OSCE como instrumento de avaliação em ginecologia e obstetrícia: percepção dos alunos de medicina da UFRN [dissertation]. Natal: Universidade Federal do Rio Grande do Norte; 2018 [access in 13 nov. 2019]. Available from: https://repositorio.ufrn.br/jspui/ handle/123456789/25853.

18. Bandura A. Social Foundations of thought and action: a social cognitive theory. New York: Englewood Cliffs, Prentice Hall; 1986.

19. laochite RT, Costa Filho RA, Matos MM, Sachimbombo KMC. Autoeficácia no campo educacional: revisão das publicações em periódicos brasileiros. Psicol Esc Educ. 2016;20(1):45-54. doi: 10.1590/2175-353920150201922.

20. Bandura A. Swimming against the mainstream: the early years from chilly tributary to transformative mainstream. Behavior Research and Therapy. 2004;42:613-30. doi: 10.1016/ j.brat.2004.02.001.

21. Moreira TC, Ambiel RAM, Nunes MFO. Escala de fontes de autoeficácia para escolha profissional: construção e estudos psicométricos iniciais. Temas Psicol. 2018;26(1):47-60. doi: 10.9788/TP2018.1-03Pt.

22. Leonardo FL, Murgo CS, Sena BCS. A ação pedagógica e a autoeficácia docente no ensino superior. EccoS - Rev Cient. 2019;0(48):255-72. doi: 10.5585/eccos.n48.8058.

23. Cordeiro GMC, Souza JRS. A importância da afetividade no processo de ensinoaprendizagem. Revista Khora. 2019;6(7) [access in 13 nov. 2019]. Available from: http://www.site.feuc.br/khora/index.php/vol/article/viewFile/165/112.

24. Freire I, Bahia S, Estrela $M$, Amaral A. A dimensão emocional da docência: contributo para a formação de professores. Revista Portuguesa de Pedagogia. 2014;46(2):151-71 [access in 23 sep. 2019]. Available from: https://impactum-journals.uc.pt/rppedagogia/article/view/1742.

25. Bahia S, Freire IP, Estrela MT, Amaral A, Espírito Santo JA. The Bologna process and the search for excellence: between rhetoric and reality, the emotional reactions of teachers. Teaching in Higher Education. 2017;22(4):467-82. doi: 10.1080/13562517.2017.1303471.

26. Megale L. Processos avaliativos no curso de medicina: desempenho dos estudantes em relação às competências em pediatria e sua significação pelo docente [thesis]. Belo Horizonte: Universidade Federal de Minas Gerais; 2011 [access in 23 sep. 2019]. Available from: http://hdl.handle. net/1843/EJAO-8JWK7Z. 
27. laochite RT. Auto-eficácia de docentes de Educação Física [thesis]. Campinas: Universidade Estadual de Campinas; 2007 [access in 20 oct. 2019]. Available from: http://repositorio.unicamp.br/jspui/handle/REPOSIP/252026.

28. Bandura A, Azzi RG, Polydoro S. Teoria Social Cognitiva: conceitos básicos. Porto Alegre: Artmed; 2008.

29. Dybowski C, Sehner S, Harendza S. Influence of motivation, self-efficacy and situational factors on the teaching quality of clinical educators. BMC Med Educ. 2017;17(1). doi: 10.1186/s12909-017-0923-2.

30. Polydoro S, Winterstein PJ, Azzi RG, Carmo AP, Venditti R. Escala de autoeficácia do professor de educação física. In: Machado C, Almeida LS, Gonçalves M, Ramalho V, coordenadores. Avaliação psicológica: formas e contextos. Braga: Psiquilíbrios; 2004. p. 330-7.

31. Tschannen-Moran M, Hoy AW. Teacher efficacy: capturing an elusive construct. Teach Teach Educ. 2001;17(7):783-805. doi: 10.1016/s0742051x(01)00036-1.

32. Bandura A. Self-efficacy: the exercise of control. New York: W. H. Freeman and Company; 1997.

33. Issenberg SB, McGaghie WC, Petrusa ER, Gordon DL, Scalese RJ. Features and uses of high-fidelity medical simulations that lead to effective learning: a BEME systematic review. Med Teach. 2005;27(1):10-28. doi: $10.1080 / 01421590500046924$
34. Dancey $\mathrm{CP}$, Reidy J. Estatística sem matemática para psicologia usando SPSS para Windows. Porto Alegre: Artes Médicas; 2006.

35. Rocha MS. A auto-eficácia docente no ensino superior [thesis]. Campinas: Universidade Estadual de Campinas; 2009 [access in 23 sep. 2019]. Available from: http://www.repositorio.unicamp.br/handle/REPOSIP/251766.

36. Azzi RG, Vieira DA, laochite RT, Ferreira LCM, Guerreiro-Casanova DC. Crenças de eficácia pessoal e coletiva. In: Azzi RG, Vieira DA. Crenças de eficácia em contexto educativo. São Paulo: Casa do Psicólogo; 2014. p. 13-24.

37. Khan KZ, Ramachandran S, Gaunt K, Pushkar P. The Objective Structured Clinical Examination (OSCE): AMEE Guide no 81. Part I: An historical and theoretical perspective. Med Teach. 2013;35(9):1437-46. doi: 10.3109/0142159x.2013.818634.

38. Ribeiro JRC, Bicalho CCF, Onofre MTAS, Noc F. Autoeficácia em professores do ensino superior. Revista de Ensino, Educação e Ciências Humanas. 2019;20(3):319-26. doi: 10.17921/2447-8733.2019v20n3p319-326. 